\title{
Early and Late Complications after Hepatic Arterial "Port-a-Cath" Implantation in the Treatment of Hepatic Metastasis from Colorectal Cancer
}

\author{
Simona Ruxandra Volovat ${ }^{1 *}$, Serban Negru ${ }^{2}$, Vasile Maciuc ${ }^{3}$ and Viorel Scripcariu \\ ${ }^{1}$ Regional Cancer Institute lasi, "Gr.T. Popa" University of Medicine and Pharmacy lasi, Romania \\ ${ }^{2}$ Department of Oncology, University of Medicine and Pharmacy Timisoara, Romania
}

"Ion lonescu de la Brad" University of Agricultural Sciences and Veterinary Medicine lasi, Romania

\begin{abstract}
Background and Aim: In metastatic colorectal cancer, in the last 10 years, hepatic arterial infusion (HAl) was proposed as an alternative using various chemotherapy agents. The insertion of a port-a-cath in the hepatic artery is needed and there are various methods to do that, from classical to interventional approach.

Patients and Methods: Patients were selected with metastatic colorectal cancer with inoperable liver metastasis only and were treated with oxaliplatin HAI, combined with systemic intravenously chemotherapy. The port-a-cath insertion was done using the classical approach in the same surgical time with the subclavicular vein port insertion.

Results: Thirty-two patients were treated. During our experience we did not encounter intra-operative complications. Among the immediate post-operative complications mainly consisted of metabolic complications $(6.2 \%)$ and infection was the most common late complication (9.4\%). In one case we removed the port-a-cath, thus the patient was not able to continue the treatment.

Conclusion: When talking about the safety of the procedure, we didn't find it to be more at risk for the patients compared to the literature. Even though the antibiotic prophylaxis is done regularly, the risk of infection remains, especially as a late complication
\end{abstract}

Keywords: Metastatic colorectal cancer; Hepatic arterial infusion; Hepatic port-a-cath implantation

\section{Background}

The colorectal cancer (CRC) still represents in the modern era a major health problem, being the second cause of cancer related mortality, despite the major scientific progresses and the emerge of many new biological agents currently used in the treatment. The liver is known to be the first metastatic site in CRC and approximately $60-70 \%$ of patients develop them during the course of the disease, out of which only $10 \%$ are eligible for a partial liver resection, the only curative treatment in this disease.

Nowadays, the modern systemic treatment with or without biological agents has the purpose to switch the patients with an unresectable liver disease to a resectable stage.

Regional hepatic arterial infusion of chemotherapy (HAI) aims to deliver higher local drug concentration to un-resectable liver tumors, leading to fewer significant systemic side effects compared to standard chemotherapy. The theoretical basis for treating liver tumors with HAI is that hepatic neoplasms receive $\sim 95 \%$ of their blood supply from the hepatic artery, unlike normal hepatocytes, which are perfused mainly by the portal vein [1]. It has been proved that patients treated with HAI have a better response rate than those on systemic chemotherapy, despite the low impact on overall survival $[2,3]$. When it is associated with systemic chemotherapy, the use of HAI could offer a better control of the extra-hepatic disease. Therefore, it remains an important treatment option in patients with advanced, inoperable primary or metastatic hepatic tumors.

A rather large experience with the HAI technique has been obtained in patients with isolated liver metastases of colorectal cancer. There are several randomized trials on HAI using FUDR or 5-FU in patients with non-resectable colorectal liver metastases that have been performed since the 1980, but achieved inconsistent results. This fact could be explained on one side by the inadequate study designs at the time and on the other side by the technical problems with the application of the devices [4-6].

This last issue was significantly improved in the last decade by introducing different techniques of placing the catheter. Most of the studies with HAI have been performed on the colorectal cancer patients with un-resectable liver metastasis [7] and not on primary liver cancer patients.

This administration of cytotoxic agents to the liver requires catheters connected to subcutaneous ports in order to allow an easy and repetitive infusion of chemotherapy directly to liver tumors. Surgically implanted hepatic catheters have shown to have considerable complication rates and the repair or replacement of the malfunctioning port systems often require further surgery procedures. The aim of this article is to provide a comprehensive review of early and late complications of surgical implanted catheter-port systems for HAIC.

In order to define the safety and the practicability of the HAI approach, we included patients with colorectal cancer and nonresectable liver metastasis in a prospective study that took place in our hospital. The patients were further included in a study that compared

*Corresponding author: Simona Ruxandra Volovat, MD, Regional Cancer Institute lasi, General Berthelot Street No. 24, 700483, lasi, Romania, Tel: +40 (0) 3742788 10; E-mail: simona v2002@yahoo.com

Received May 10, 2014; Accepted July 15, 2014; Published December 20, 2014

Citation: Volovat SR, Negru S, Maciuc V, Scripcariu V. Early and Late Complications after Hepatic Arterial "Port-a-Cath" Implantation in the Treatment of Hepatic Metastasis from Colorectal Cancer. Journal of Surgery [Jurnalul de chirurgie] 2015; 10(4): 277-281 DOI:10.7438/1584-9341-10-4-7

Copyright: (c) 2015 Volovat SR, et al. This is an open-access article distributed under the terms of the Creative Commons Attribution License, which permits unrestricted use, distribution, and reproduction in any medium, provided the original author and source are credited. 
the standard chemotherapy regimen FOLFIRI with FOLFIRI associated with the local administration of Oxaliplatin using the hepatic inserted port. The primary objectives of the study were progression free survival; among the secondary objectives, which will be detailed in this article, are "complication rates" and "safety of the device and regional therapy".

\section{Material and Method}

\section{Study Design, Patient's Collective and Eligibility Criteria}

Patients with colorectal cancer with non-resectable liver metastasis were enrolled into a prospective phase II study that aimed to compare the administration of FOLFIRI vs. FOLFIRI and Oxaliplatin administered in the hepatic artery. The FOLFIRI regimen consisted of irinotecan $160 \mathrm{mg} \mathrm{m}^{-2} \mathrm{IV}$ in 90 minutes, leucovorin $200 \mathrm{mg} \mathrm{m}^{-2} \mathrm{IV}$ in 90 minutes (concurrently with leucovorin in separate bags via $y$-line connection) and 5-FU $2400 \mathrm{mg} \mathrm{m}^{-2}$ in 48 hours (with an ambulatory elastomeric pump), repeated at 14 days. Oxaliplatin was administered $85 \mathrm{mg} \mathrm{m}^{-2}$ infused in day 1 over 2 hours, combined with systemic chemotherapy. Apart from the primary endpoints like progression free survival and response rate, we also considered evaluating the technical complications and safety associated with the use of implanted port catheter systems. The study was performed from 2011 to 2013. The local ethics committee of the University of Medicine and Pharmacy Iasi, Romania and Victoria Hospital Iasi, Romania approved the protocol, and a detailed written informed consent was obtained from every patient prior to treatment.

The patients included in the trial met the following inclusion criterias:

- Age over 18 years-old;

- Proven histology of colorectal adenocarcinoma

- Stage IV colorectal cancer,

- Eastern Cooperative Oncology Group (ECOG) performance status $0-2$

- Estimated life expectancy of $\geq 3$ months.

Leukocyte count of at least $3.5 \times 109 / \mathrm{L}$, neutrophils count of at least $1.5 \times 109 / \mathrm{L}$, platelet count of at least 100X109/L, satisfactory biochemical results (serum creatinine of $1.3 \mathrm{mg} / \mathrm{dL}$ or less, serum bilirubin less than $1.5 \mathrm{mg} / \mathrm{dL})$

Measurable disease (hepatic lesion with longest diameter of minimum $20 \mathrm{~mm}$ ) according to RECIST 1.1 criteria with liver invasion assessed by computed tomography (CT) as less than $50 \%$ of the liver.

The main exclusion criteria were:

- Detectable extra-hepatic disease (including during laparotomy for catheterization of hepatic artery)

- Hepatic artery thrombosis,

- Peripheral neuropathy,

- Active infections,

- Inflammatory bowel disease,

- Total colectomy,

- Heart failure,

- Respiratory insufficiency,

- Severe coronary artery disease or failure of other organs.

Previous adjuvant chemotherapy including irinotecan or oxaliplatin were excluded, but previous fluoropyrimidine-based adjuvant chemotherapy was allowed if it ended more than 6 months before random assignment.

We have subcutaneously placed hepatic arterial ports in our Surgical Unit on 32 patients aged between 37 and 75 years old, which were eligible for the protocol of systemic chemotherapy with FOLFIRI and hepatic arterial infusion with Oxaliplatin. Most of the patients had no prior chemotherapy for metastatic disease, but some had adjuvant chemotherapy before becoming metastatic, but neither had chemotherapy less than 6 months prior to entering the study.

The protocol involved also the placement of a subclavian catheter in order to administer the systemic chemotherapy that consisted in FOLFIRI protocol during a longer period of time and with better tolerance for the patients.

\section{Interventions-Port Implantation Procedure}

After identifying the right gastroepiploic artery, using as reference the proximity to the inferior margins of the duodenal bulb, we continue with the careful dissection of the artery from the vein located in the immediate vicinity. The right gastroepiploic artery at this level has a caliber that will allow the catheterization of the gastro-duodenal artery and then of the hepatic artery. After the distal ligature of the right gastroepiploic artery we can puncture the artery in the proximal segment and an arteriography can be made in order to visualize the hepatic artery and its entire course (Figure 1). This arteriography is not a compulsory maneuver because it will be accomplished after placing the catheter.

A small transversal incision of the right wall of the gastroepiploic artery is made and the guide wire is introduced (Teflon guide wire 0.35 "), which ascends afterwards by pushing it into the hepatic artery, passing through the gastro-duodenal artery. At this moment, palpating the hepatic artery in the hepatic pediculum in the same time with ascending the guide wire will allow us to place the catheter properly.

Afterwards, we proceed to the insertion of the silicon radio-opaque catheter in the proper hepatic artery and ascending as much as possible in the hepatic artery towards the hepatic hilum, carefully feeling by hand the artery in the hepatic pediculum (Figures 2-4). During this step or in the moment of the introduction of the guide wire, it may be possible that there is the tendency to engage towards the celiac trunk through the common hepatic artery. If this happens, the guide wire or the catheter is retracted and we try to re-position it.

At this point, after the correct positioning, a hepatic arteriography is made through the introduced catheter in order to verify its position.

After the correct positioning of the catheter in the hepatic artery we will proceed to the chamber positioning (capsule, the "infusional

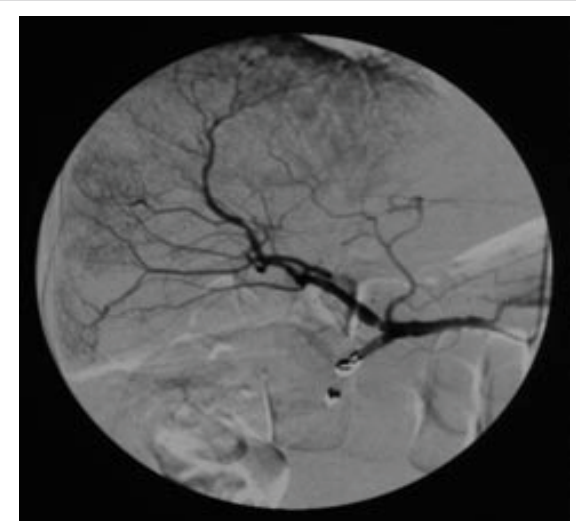

Figure 1: The Arteriography made Indicates the Correct Position of the Catheter in the Common Hepatic Artery. 


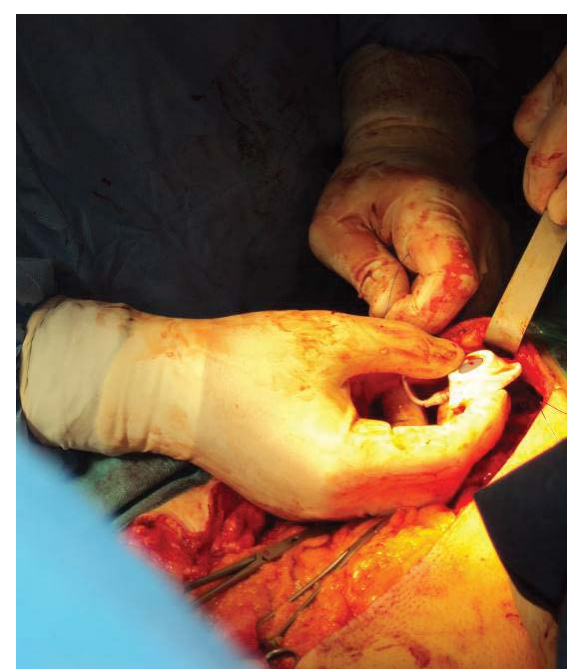

Figure 2: Port-a-Cath Insertion in the Hepatic Artery.

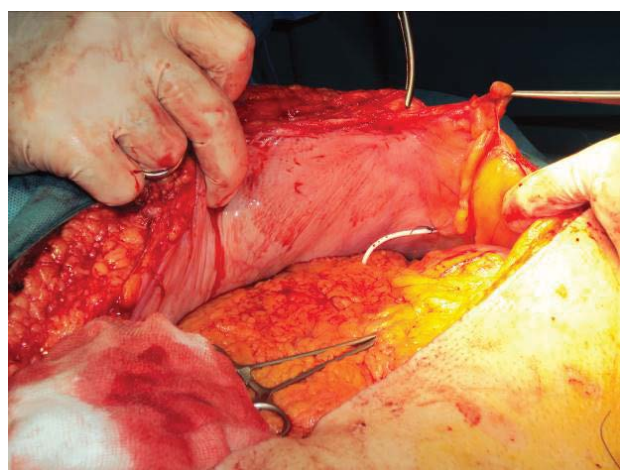

Figure 3: Port-a-Cath Insertion in the Hepatic Artery.

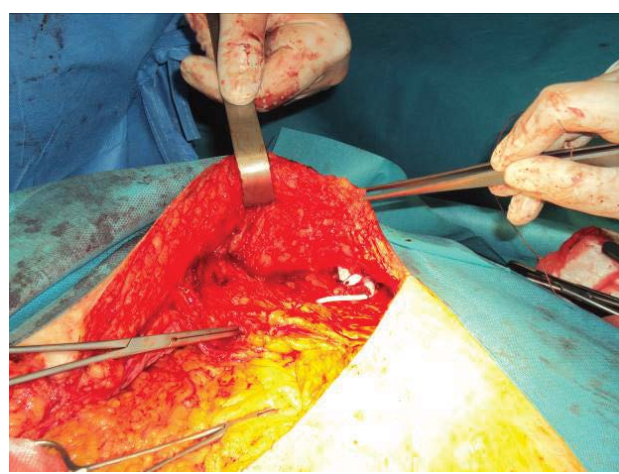

Figure 4: Port-a-Cath Insertion in the Hepatic Artery.

port") on the inferior part of the right rib cage on the same layer as the medial-clavicular line, taking into consideration the fact that the entire capsule should be placed on the hard layer of the last ribs of the rib cage. In order to accomplish this separate skin incision made, at approximately $3-5 \mathrm{~cm}$ proximal to the chamber's positioning spot and the dissection of the subcutaneous layer to the muscular-aponeurotic layer of the costal margin of the last ribs. This dissection is done distally of the incision, in order to avoid the over-positioning of the incision, and so of the future scar, over the spot of the future injections of the capsule. Through dissection, a subcutaneous pocket is created in which we will place the capsule and fix it with three suture points to the muscular-aponeurotic layer. The maneuver is necessary in order to avoid a possible migration or subcutaneous torsion of the capsule, thus being impossible to use it for injections in the future.
Afterwards a subcutaneous tunnel is being created to the peritoneal cavity, using the metallic perforator from the port-a-cath's kit and the already positioned in the hepatic artery catheter is being brought through this subcutaneous tunnel right to the proximity of the capsule. Using sealing fixing elements of the catheter to the port-a-cath's capsule, the catheter is being connected to the chamber. The physical functionality is being verified and also using arteriography, after which, the procedure is being considered closed.

\section{Surgical Incidents and Accidents}

The impossibility to identify the right gastroepiploic artery in a well-represented fat tissue covering the epiplones. The dissection is being made in a more proximal position, closer to the gastro-duodenal artery.

A hematoma situated at the dissection site can be an impediment in appreciating the anatomy and the trajectory of the right gastroepiploic artery to the emerging point from the gastro-duodenal artery. The dissection is made in a more proximal position, closer to the gastroduodenal artery.

Guide wire or catheter malposition towards the celiac trunk. The positioning procedure is retried and an arteriographic control for an eventual anatomic variant is done.

The malposition of the capsule in the thickness of the abdominal wall and not on the hard layer of the costal margin. Another subcutaneous pocket will be made.

A bleed of a hematoma where the capsule is situated. The hemostasis must be carefully controlled in order to avoid the hematoma, which can be a source of infection for the capsule.

\section{Late Complications}

Infection where the subcutaneous capsule is located. It is a severe complication and antibiotic treatment according to the antibiogram is needed. The repositioning of the capsule or even the removal of the port-a-cath is compulsory. The repositioning of the port-a-cath is made under large-spectrum antibiotics administered in prophylaxis, according to the existent protocols for a surgical procedure.

Port-a-cath malfunction and its blockage through blood cloth obstruction. We try a de-obstruction or, if not, the port-a-cath is suppressed. This incident must be avoided by a proper maintenance with heparin after the procedure or after the chemotherapy, once a week or in between administrations.

In the same time with placing the catheter in the hepatic artery, a port-a-cath in the subclavian or jugular vein can be placed, in order to be able to administer the concomitant systemic chemotherapy. The technique is the classic Seldinger technique, percutaneously, by finding the subclavian or jugular vein and placing the capsule in a prepectoral subcutaneous pocket. The radiologic control is necessary after positioning the catheter, in order to avoid the complications due to the catheter malposition.

\section{Evaluations}

\section{Pretreatment Evaluation and Follow-up}

We recorded each patient's history; clinical examination and routine laboratory status were performed at least 10 days before the first chemotherapy application. Basic imaging consisted of an abdominal CT, chest-X-ray or CT. Before every HAI-application a clinical evaluation, a full blood count and chemistry were repeated. Assessment of toxicity was performed according to WHO-criteria. Response evaluation was performed according to RECIST 1.1 criteria and repeated in 3-monthly intervals using CT evaluations. In case of progression (end of treatment), patients were seen at least every 
3 months. The treatment was interrupted in case of grade IV WHO toxicity, irreversible loss of port function or disease progression.

\section{Port Complications}

Port-related adverse events were assessed from the date of implantation. The duration was defined as functional device with or without revision, but without the requirement for removing the entire system.

\section{Statistical Evaluations}

Our data was analyzed by using SPSS 16.00 for Windows. We determined the frequency, the regression coefficient, Pearson correlation and relative risk (RR) assessment. We also used in our study Chi-Square tests and "p" significance test. A two-sided p-value less than 0.05 were considered to prove significance for all tests performed.

\section{Results}

\section{Patient's Characteristics}

During 2011-2013 a number of 32 patients underwent the surgical implantation of port-a-cath, as described, in the subclavian vein and hepatic artery. The patients were aged between 37 and 75 years old, but with a higher age frequency between 51-70 years old $(n=21)$. $71.9 \%(\mathrm{n}=23)$ were diagnosed with rectal adenocarcinoma, $15.6 \%$ $(n=5)$ were colon adenocarcinoma, $9.4 \%(n=3)$ had double colo-rectal localization and one patient had a different histology (neuroendocrine tumor) after resection. Most patients had a well-differentiated (grade 2) adenocarcinoma $(46.9 \%, n=15)$, only $25 \%(n=8)$ being undifferentiated.

In our lot of patients $6.2 \%(\mathrm{n}=2)$ had a unique hepatic lesion, unresectable due the large dimension $(>5 \mathrm{~cm})$ or due to the proximity with large vessels. In most cases $(56.2 \%, \mathrm{n}=18)$, our patients had multiple hepatic lesions between $2-5 \mathrm{~cm}$. 84.4\% (27) of the patients had synchronous hepatic metastasis and in these cases the primary tumor was resected at the same time with implanting the port-a-cath. A large proportion $(65.6 \%, n=21)$ hadn't had previous chemotherapy for metastatic disease (especially irinotecan or oxaliplatin based chemotherapy), but some patients with rectal cancer had previous perioperative radio-chemotherapy with capecitabine, ended more than 6 months before random assignment. (Table I)

\section{Complications}

During our study, all patients received peri-operative antibiotic treatment for 7 days according to internal guidelines. During the surgery, we did not encounter any intraoperative complications. The immediate port-related complications mainly consisted of metabolic complications, post-surgery pre-renal insufficiency, which had a fast response to medical treatment. Infectious problems (such as sepsis) and also fistulae were less frequent (only one case) and were resolved without compromising the oncological treatment (Table II).

Late complications were mainly infectious $(9.4 \%, n=3)$, which needed hospitalization and complex antibiotic treatment, as all patients were at the moment under chemotherapy, thus being at a higher infectious risk due to post-chemotherapy induced neutropenia. In all cases, all complications were resolved. In one case, a patient had a port-a-cath infection that obliged us to remove it (Figure 5). From that point on, the patient did no longer receive the HAI perfusions with oxaliplatin.

\section{Discussion}

The oncological patients sometimes need long-term systemic or local chemotherapy, which can be achieved through an access system connected to an access port. An implantable device, which consists of a reservoir, a capsule connected to a catheter, will allow the continuous access for the treatment and can lead to an increased quality of life for the patients.

Among different techniques used in order to insert a port-a-cath into the hepatic artery [8-10], the fixed catheter tip method [11] has proven the advantage of preventing catheter dislocation and hepatic artery obstruction, thus being used in most centers performing this technique.

A review of large studies involving the percutaneous placement of port-a-cath for HAI administration using the fixed-catheter method described a rate of catheter dislocation between 2-8\% [1,12].

The present study evaluated a heterogeneous group of 32 patients diagnosed with metastatic colorectal adenocarcinoma limited to the liver. For these patients a curative approach of the hepatic involvement was not feasible due to the number ( $>2$ lesions), size $(>2-5 \mathrm{~cm})$ or localization (the unique lesions were located close to major vessels and, as a consequence, metastasectomy could not be performed). In these cases we opted for an interventional approach in order to obtain a better local response and control. A protocol consisting of systemic chemotherapy by FOLFIRI protocol and an intra-arterial

Table I: Patient's Characteristics.

\begin{tabular}{|c|c|}
\hline Patient's characteristic & $\mathbf{N}(\%)$ \\
\hline \multicolumn{2}{|l|}{ Sex } \\
\hline Male & $16(50)$ \\
\hline Female & $16(50)$ \\
\hline \multicolumn{2}{|l|}{ Age at diagnosis } \\
\hline$<50$ years-old & $9(28.1)$ \\
\hline $51-70$ years-old & $21(65.6)$ \\
\hline$>70$ years-old & $2(6.2)$ \\
\hline \multicolumn{2}{|l|}{ Histology } \\
\hline Colon adenocarcinoma & $5(15.6)$ \\
\hline Rectal adenocarcinoma & $23(71.9)$ \\
\hline Double localization (colon and rectum) & $3(9.4)$ \\
\hline \multicolumn{2}{|l|}{ Tumor grade } \\
\hline Poorly-differentiated & $8(25)$ \\
\hline Moderately-differentiated & $1(3.1)$ \\
\hline Well-differentiated & $15(46.9)$ \\
\hline $\mathrm{N} / \mathrm{A}$ & $8(25)$ \\
\hline \multicolumn{2}{|l|}{ Hepatic lesion(s) } \\
\hline Unique lesion $>5 \mathrm{~cm}$ & $2(6.2)$ \\
\hline Multiple lesions $>5 \mathrm{~cm}$ & $9(28.1)$ \\
\hline Multiple lesions $2-5 \mathrm{~cm}$ & $18(56.2)$ \\
\hline Multiple lesions $<2 \mathrm{~cm}$ & $3(9.4)$ \\
\hline \multicolumn{2}{|l|}{ Metastasis } \\
\hline Synchronous & $27(84.4)$ \\
\hline Metachronous & $5(15.6)$ \\
\hline \multicolumn{2}{|l|}{ Previous chemotherapy } \\
\hline Yes & $11(34.4)$ \\
\hline No & $21(65.6)$ \\
\hline
\end{tabular}

Table II: Intra and Postoperative Complications.

\begin{tabular}{|c|c|}
\hline Complications & $\mathbf{N}(\%)$ \\
\hline Intra-operative complications & $0(0)$ \\
\hline Early surgical complications & $28(87.5)$ \\
\hline None & $2(6.2)$ \\
\hline Metabolic complications & $1(3.1)$ \\
\hline Infectious complications & $1(3.1)$ \\
\hline Fistulae & \\
\hline Late surgical complications & $28(87.5)$ \\
\hline None & $3(9.4)$ \\
\hline Infectious complications & $1(3.1)$ \\
\hline Dislocation of catheter's chamber & \\
\hline
\end{tabular}




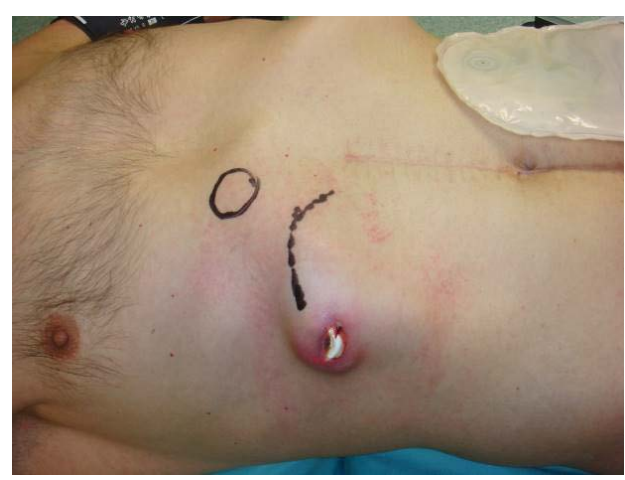

Figure 5: Infected Port-a-Cath that needed to be Removed.

administration in the hepatic artery of Oxaliplatin was implemented for these patients. In the present study, among the primary objectives such as response rate and progression free survival, we also considered secondary objectives such as toxicity profiles, safety and, in some cases, the target was the ulterior possibility of resectability of the hepatic metastasis in case of a good response to chemotherapy. The present paper addresses one of the secondary objectives that of the safety of the procedure, taking into account the fact that it is not a standardized treatment option for these patients.

It was found that the implantation of port-a-cath in the hepatic artery was feasible in all patients, and that the rate of device relatedcomplications was relatively low. In addition, the proportion of patients with treatment discontinuation due to port-failure compares favourably with the literature $[13,14]$ (only one patient out of 32).

In our study, the rate of catheter dislocation was comparable with the data from the literature (3.1\%) and the correction was possible without further complications. Even though other studies mention the difficulty in catheter removal as a major issue, we had a $100 \%$ rate of success. Tajima et al. describes in his study a withdrawal method of the port-a-cath implanted using the fixed-tip method [15]. He reported several cases of catheter removal due to infection, catheter obstruction, hepatic artery obstruction or catheter dislocation and 14 of 15 cases were successful and 10 out of 15 patients underwent reinsertion of the port-catheter system. He also developed a new system called the selfretaining indwelling catheter system that required a special device.

In this study, the percutaneous positioning of arterial port is a safe and effective technique to deliver loco-regional treatment for hepatic tumors. Complication rates are similar to literature reports, being in an acceptable range. Additionally, the occurrence of infectious complications or chamber dislocation was low and the difficulty of removal was rare. In conclusion, this percutaneous approach in the hands of a skilled team allows intra-arterial infusion in a wide percentage of patients.

\section{Conclusions}

The insertion of a port-a-cath in the hepatic artery remains an experimental procedure, with limited indications. However, we find this procedure to be more and more used in clinical trials or as part of a local treatment of the hepatic metastasis.

Although there are multiple techniques described in the literature, we prefer the fixed catheter tip method due to its advantages and to the fact that it has been widely utilized.

In our study, we can conclude that the insertion of the port-a-cath into the hepatic artery is a safe procedure, with limited complications during or after surgery. Moreover, these complications were manageable in all cases and this percutaneous approach, in the hands of a skilled team, allows intra-arterial infusion in a wide percentage of patients.

\section{Conflict of interests}

Authors have no conflict of interests to disclose

\section{Acknowledgement}

The first author, Simona Ruxandra Volovat is PhD student of "Gr.T. Popa" University of Medicine and Pharmacy lasi, Romania. The present study is the result of research program during $\mathrm{PhD}$ thesis studentship.

\section{References}

1. Breedis C, Young G (1954) The blood supply of neoplasms in the liver. Am J Pathol 30: 969-977.

2. Cohen AM, Kemeny NE, Kohne CH, , Wils J, de Takats PG, et al. (1996) Is intraarterial chemotherapy worthwhile in the treatment of patients with unresectable hepatic colorectal cancer metastases? Eur J Cancer 32A: 2195-2205.

3. Goldberg JA, Kerr DJ, Stewart I, McArdle CS (1990) A comparison of regional and systemic chemotherapy for hepatic metastases. Eur J Surg Oncol 16: 464467.

4. Kingham TP, D'Angelica M, Kemeny NE (2010) Role of intra-arterial hepatic chemotherapy in the treatment of colorectal cancer metastases. J Surg Oncol 102: 988-995

5. Mocellin S, Pilati P, Lise M, Nitti D (2007) Meta-analysis of hepatic arterial infusion for unresectable liver metastases from colorectal cancer: the end of an era? J Clin Oncol 25: 5649-5654.

6. Bouchahda M, Lévi F, Adam R, Rougier P (2011) Modern insights into hepatic arterial infusion for liver metastases from colorectal cancer. Eur $\mathrm{J}$ Cancer 47: 2681-2690.

7. Hildebrandt B, Pech M, Nicolaou A, Langrehr JM, Kurcz J, et al. (2007) Interventionally implanted port catheter systems for hepatic arterial infusion of chemotherapy in patients with colorectal liver metastases: a Phase II-study and historical comparison with the surgical approach. BMC Cancer 7:69.

8. Wacker FK, Boese-Landgraf J, Wagner A, Albrecht D, Wolf KJ, et al. (1997) Minimally invasive catheter implantation for regional chemotherapy of the liver: a new percutaneous transsubclavian approach. Cardiovasc Intervent Radiol 20: $128-132$.

9. Clouse ME, Ahmed R, Ryan RB, Oberfield RA, McCaffrey JA (1977) Complications of long term transbrachial hepatic arterial infusion chemotherapy. AJR Am J Roentgenol 129: 799-803.

10. Tanaka T, Arai Y, Inaba Y, Matsueda K, Aramaki T, et al. (2003) Radiologic placement of side-hole catheter whih tip fixation for hepatic arterial infusion chemotherapy. J Vasc Interv Radiol 14: 63-68.

11. Yamagami T, Nakamura T, Yamazaki Y, lida S, Kato T, et al. (2002) Cathetertip fixation of a percutaneously implanted port-catheter system to prevent dislocation. Eur Radiol 12: 443-449.

12. Chen Y, He X, Chen W, Lu W, Mei Q, et al. (2000) Percutaneous implantation of a Port-Catheter System using the left subclavian artery. Cardiovasc InterventRadiol 23: 22-25.

13. Tanaka T, Arai Y, Inaba Y, Matsueda K, Aramaki T, et al. (2003) Radiologic placement of side-hole catheter with $t$ fixation for hepatic arterial infusion chemotherapy. J Vasc Interv Radiol 14: 63-68.

14. Sinn M, Nicolaou A, Ricke J, Podrabsky P, Seehofer D, et al. (2013) Interventionally implanted port catheter systems for hepatic arterial infusion of chemotherapy in patients with primary liver cancer: A phase II-study (NCT00356161). BMC Gastroenterology 13: 125.

15. Yamagami T, Kato T, Yoshimatsu R, Matsumoto T, Nishimura T (2006) Withdrawal of port-catheter system for hepatic arterial infusion chemotherapy implanted with fixed catheter tip method. J Vasc Interv Radiol 17: 651-656. 